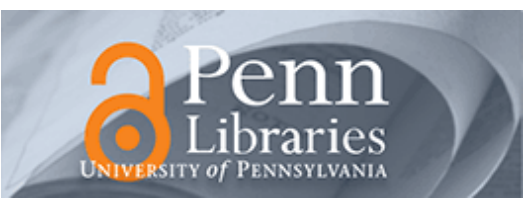

University of Pennsylvania

ScholarlyCommons

September 2003

\title{
Towards a factored analysis of legged locomotion models
}

\author{
Richard Altendorfer \\ University of Michigan \\ Daniel E. Koditschek \\ University of Pennsylvania, kod@seas.upenn.edu \\ Philip Holmes \\ Princeton University
}

Follow this and additional works at: https://repository.upenn.edu/ese_papers

\section{Recommended Citation}

Richard Altendorfer, Daniel E. Koditschek, and Philip Holmes, "Towards a factored analysis of legged locomotion models", . September 2003.

Copyright 2003 IEEE. Reprinted from Proceedings of the 2003 IEEE International Conference on Robotics and Automation (ICRA 2003), Volume 1, pages 37-44.

This material is posted here with permission of the IEEE. Such permission of the IEEE does not in any way imply IEEE endorsement of any of the University of Pennsylvania's products or services. Internal or personal use of this material is permitted. However, permission to reprint/republish this material for advertising or promotional purposes or for creating new collective works for resale or redistribution must be obtained from the IEEE by writing to pubs-permissions@ieee.org. By choosing to view this document, you agree to all provisions of the copyright laws protecting it.

NOTE: At the time of publication, author Daniel Koditschek was affiliated with the University of Michigan. Currently (August 2005), he is a faculty member in the Department of Electrical and Systems Engineering at the University of Pennsylvania.

This paper is posted at ScholarlyCommons. https://repository.upenn.edu/ese_papers/125

For more information, please contact repository@pobox.upenn.edu. 


\title{
Towards a factored analysis of legged locomotion models
}

\author{
Abstract \\ In this paper, we report on a new stability analysis for hybrid legged locomotion systems based on \\ factorization of return maps. We apply this analysis to a family of models of the Spring Loaded Inverted \\ Pendulum (SLIP) with different leg recirculation strategies. We obtain a necessary condition for the \\ asymptotic stability of those models, which is formulated as an exact algebraic expression despite the \\ non-integrability of the SLIP dynamics. We outline the application of this analysis to other models of \\ legged locomotion and its importance for the stability of legged robots and animals.
}

\section{Comments}

Copyright 2003 IEEE. Reprinted from Proceedings of the 2003 IEEE International Conference on Robotics and Automation (ICRA 2003), Volume 1, pages 37-44.

This material is posted here with permission of the IEEE. Such permission of the IEEE does not in any way imply IEEE endorsement of any of the University of Pennsylvania's products or services. Internal or personal use of this material is permitted. However, permission to reprint/republish this material for advertising or promotional purposes or for creating new collective works for resale or redistribution must be obtained from the IEEE by writing to pubs-permissions@ieee.org. By choosing to view this document, you agree to all provisions of the copyright laws protecting it.

NOTE: At the time of publication, author Daniel Koditschek was affiliated with the University of Michigan. Currently (August 2005), he is a faculty member in the Department of Electrical and Systems Engineering at the University of Pennsylvania. 
Proceedings of the 2003 IEEE

International Conference on Robotics \& Automation

Taipei, Taiwan, September 14-19, 2003

\section{Towards a factored analysis of legged locomotion models}

\author{
Richard Altendorfer, Daniel E. Koditschek \\ Dept. of Electrical Engineering and Computer \\ Science, \\ The University of Michigan, Ann Arbor, MI \\ 48109, USA
}

\begin{abstract}
In this paper, we report on a new stability analysis for hybrid legged locomotion systems based on factorization of return maps. We apply this analysis to a family of models of the Spring Loaded Inverted Pendulum (SLIP) with different leg recirculation strategies. We obtain a necessary condition for the asymptotic stability of those models, which is formulated as an exact algebraic expression despite the non-integrability of the SLIP dynamics. We outline the application of this analysis to other models of legged locomotion and its importance for the stability of legged robots and animals.
\end{abstract}

\section{INTRODUCTION}

This paper introduces a new formalism for studying the stability of legged locomotion gaits and other periodic dynamically dexterous robotic tasks. We are motivated by the need to explain and control the remarkable performance of RHex, an autonomous hexapedal robot runner with unparalleled mobility [1]. Powered by only six actuators, located at the "hips" to drive each of its six passively compliant legs, RHex's locomotion is excited by a single periodic "clock" signal split into phase and anti-phase copies for coordinating its altemating tripod gait. A simple PD controller at each hip motor in a given tripod forces its leg to track the alternately fast and slow clock reference signal corresponding to putative stance and swing phases. RHex's stable gaits in varied terrain strongly motivate the development of an analytical understanding of the relationship between clock signal and steady state gait properties in this "simple" open loop case.

A complete account of this relationship in even the simple case would entail insight into the steady state properties of an under-actuated high degree of freedom hybrid mechanical system whose Lagrangian dynamics switches between a set of $2^{6}$ possible holonomically constrained models depending upon which toes are in contact with the ground. Fortunately, a growing body of simulation study and empirical evidence [2] suggests that RHex, when properly tuned, exhibits sagittal plane stance behavior well approximated by the two degree of freedom SLIP. Thus, in the short term, we seek to understand how adjustments to a coordinating clock signal will determine the steady state performance of the bipedal SLIP underlying the alternating tripod gait. In this paper, we develop the mathematical foundations of a new formalism for distinguishing volume-preserving from non-

\author{
Philip Holmes \\ Dept. of Mechanical and Aerospace \\ Engineering, \\ Princeton University, Princeton, NJ 08544, USA
}

volume-preserving hybrid Lagrangian systems. We apply this formalism to the hybrid SLIP model, the results of which suggest new insight into the relationship between clock excitation and steady state gait.

\section{A. Leg Swing Policies and Self Stability in the SLIP Template}

The SLIP model provides a ubiquitous description of biological runners in the sagittal plane [3] and, as mentioned above, a broadly useful prescription for legged robot runners such as RHex [4], [1], [2] as well. The closely related three degree of freedom Lateral Leg Spring (LLS), has been recently identified as a candidate template for cockroach running in the horizontal plane [5], [6] and seems likely to be relevant for RHex as well [1]. For present purposes, the most important insight from these models has been to provide a mathematical explanation for their unexpected "self-stability" properties (asymptotically stable equilibrium gaits in the absence of any extemal sensor based feedback inputs).

The originally discovered self-stability of SLIP [7], [8] arises from a simple leg swing policy that specifies a constant leg touchdown angle at the end of each aerial phase. A similar strategy was earlier shown to yield stable gaits in the LLS model [6]. Recently, a different timedependent leg retraction policy has been shown to inherit the stability properties of the fixed touchdown angle policy while increasing the robustness of the SLIP system [9]. On the other hand, numerical simulations of a recirculation policy where the SLIP's leg starts recirculating after leg liftoff at a constant angular velocity until leg touchdown suggest not asymptotic but neutral stability (see also [10]). Hence the leg swing policy seems to play a central role in the stability of those low-dimensional models, as is also suggested by high dimensional systems such as RHex [1] or, as established in an independently conceived model inspired by animal locomotion strategies, for a quadrupedal trotter [11].

The stability of such systems is established by reasoning about the local properties of the full-stride return map the function relating body state at one stride to body state at next - which summarizes all properties relevant to the goal of translating the body center of mass. Unfortunately, even the simplest 2dof SLIP system is non-integrable [12], 
which precludes a closed form representation of the return map that might illuminate the stability mechanisms. In this paper we will show how the stability of hybrid systems possessing certain symmetries can be analyzed in terms of their non-hybrid components, e.g. flight phase and stance phase in the case of SLIP, thus decomposing the hybrid retum map into "partial" return map factors that might be analyzed more easily.

Before doing so, we first introduce certain terminology and notation used throughout the paper by way of reviewing Liouville's theorem (see e.g. [13]), pointing out that the classical result should not be expected to apply in the present case.

\section{B. Liouville's theorem and stability}

Liouville's theorem states that volume in phase space of a holonomically constrained conservative dynamical system described by a single Hamiltonian flow is preserved, i.e. a set of initial conditions at $t=t_{0}$ in phase space will be mapped to a set with identical symplectic volume for any $t \geq t_{0}$.

Non-holonomically constrained conservative systems are in general not Hamiltonian and Liouville's theorem cannot be applied (see e.g. the asymptotic stability of the Chaplygin sleigh in [14] and references therein).

In the case of piecewise-defined holonomically constrained conservative Hamiltonian systems with different flows $f_{\alpha}^{t}$ (with $\alpha$ in an indexing set $I$ ) the transition to a new Hamiltonian flow is triggered by so-called threshold functions (for a general definition of hybrid systems see [15]). In almost all settings within robotics, these threshold functions between different flows $f_{\alpha}^{t}$ and $f_{\beta}^{t}$ depend upon state, and often have no explicit time dependence at all. Examples include a discrete version of the Chaplygin sleigh [14], [16] and low-dimensional models of legged locomotion in the horizontal and sagittal plane [6], [7], [8], which all exhibit partial asymptotic stability for certain parameter settings. Here, the (local) asymptotic stability of those hybrid system at a fixed point means that the eigenvalues of the components of their linearized return maps defined by Poincaré sections lie within the unit circle (excluding those corresponding to conserved quantities).

In those cases, the return map $\mathscr{R}$ defined by a Poincaré section is composed of several "factor" maps $r_{\alpha}^{\beta}$ that relate the state variables directly after one transition to those directly before the next. Additional transition mappings $\mathscr{T}_{\alpha}^{\beta}$ are used at transitions from flows $f_{\alpha}^{t}$ to $f_{\beta}^{t}$. They arise in practice from the fact that different flows are most easily handled analytically in different coordinate systems. Thus the return map can be decomposed into factors $\mathscr{R}=\mathscr{T}_{\gamma}^{\alpha} \circ r_{\gamma}^{\alpha} \circ \cdots \circ \mathscr{T}_{\alpha}^{\beta} \circ r_{\alpha}^{\beta}$. Liouville's theorem is not applicable to return maps where the Poincaré section is defined by the vanishing of a threshold function that is not exclusively time-dependent, since the underlying
Hamiltonian flow will map an open set of initial values to an open set on the Poincaré section with different evolution times.

\section{Contribution of this paper}

In this paper, we focus on the role of volume preservation in flows and transition maps of models of legged locomotion as an indicator of local stability. Although we cannot invoke Liouville's theorem for reasons just reviewed, we nevertheless deduce the necessity for volume preservation at a given fixed point in these flows, manifesting itself as the condition of unity determinant in the associated linearized return map. In general, in order to check whether the return map of a hybrid system $\mathscr{R}$ is volume-preserving at a fixed point, the map must be computed explicitly [16], [6], [7], [8]. However, we will show that if all vector fields $f_{\alpha}, \alpha \in I$ possess time reversing symmetries $\mathscr{S}_{\alpha}$ and if the periodic orbit giving rise to a fixed point of $\mathscr{R}$ is composed of pseudosymmetric orbits (to be defined below) on each flow domain $V_{\alpha}$, then volume-preservation of the whole system can be determined by volume-preservation on individual flow domains. Moreover, if the transition function, $h_{\alpha}^{\beta}$, from a flow domain, $V_{\alpha}$, to a next flow domain, $V_{\beta}$, enjoys a certain symmetry related to the vector field's time reversing symmetry $\mathscr{S}_{\alpha}$, then volume preservation on $V_{\alpha}$ can be determined in certain cases without an explicit expression for the flow map on that domain.

This paper introduces the rigorous foundations for the formalism just outlined, and provides an example of its value for robotics by application to the LLS [6] and SLIP [7], [8] models. As explained above, the question of whether a hybrid system is volume preserving or not has immediate consequences for gait stability. Since our conditions for volume preservation of the full stride retum map may be checked with respect to a simple "factor map," we are able to study the effects of a broad range of leg swing policies that are implementable in the analytically tractable "flight" phase of the leg. Specifically, we show how the volume preserving properties of SLIP under different leg recirculation strategies can be determined by simple differentiation. Notably, in Sec.III.A.3 we give for the first time necessary conditions for asymptotic and neutral stability of a RHex-like leg recirculation scheme applied to the SLIP model, without explicit computation of the stance phase flow map.

\section{FACTORING RETURN MAPS WITH TIME REVERSING SYMMETRIES}

Assume a hybrid mechanical system whose time evolution is described by holonomically constrained autonomous conservative vector fields $f_{\alpha}, \alpha \in I$ with configuration space variables $q_{\alpha}: \dot{x}_{\alpha}=f_{\alpha}\left(x_{\alpha}\right)$ with $x_{\alpha}=$ $\left(\begin{array}{ll}q_{\alpha} & \dot{q}_{\alpha}\end{array}\right)^{\top} \in V_{\alpha}$. The open flow domains $V_{\alpha}$ are called 
charts [15]. Transitions between vector fields are governed by threshold functions $h_{\alpha}^{\beta}$ which can depend on the initial condition $x_{\alpha_{0}}=x_{\alpha}(t=0) \in V_{\alpha}$, time $t$, and the current state $f_{\alpha}^{t}\left(x_{\alpha_{0}}\right)$. We require that for each chart there is only one threshold function $h_{\alpha}^{\beta}$. Transitions to the vector field $f_{\beta}$ are uniquely defined by $t_{\alpha_{0}}^{\beta}\left(x_{\alpha_{0}}\right)=\min _{t>0}\{t$ : $\left.h_{\alpha}^{\beta}\left(f_{\alpha}^{t}\left(x_{\alpha_{0}}\right), x_{\alpha_{0}}, t\right)=0\right\}$. In addition, all transition mappings $\mathscr{T}_{\alpha}^{\beta}$ are assumed to be volume preserving. The flow map $r_{\alpha}^{\beta}$ for the $\alpha$ th vectorfield is implicitly defined via $t_{\alpha_{0}}^{\beta}$ by $r_{\alpha}^{\beta}: x_{\alpha_{0}} \mapsto f_{\alpha}^{t_{0}^{\beta}}\left(x_{\alpha_{0}}\right)$, where $x_{\alpha_{0}}$ is assumed to be the result of a preceding chart transition.

We will define a partial return map for evolutions on individual charts $V_{\alpha}$. Under the assumption that a periodic orbit of the whole dynamical system is composed of pseudo-symmetric orbits (to be defined below) on individual charts, volume preservation of the full return map $\mathscr{R}$ can be determined in some cases without explicitly computing $r_{\alpha}^{\beta}$. This will be the case if the vector field $f_{\alpha}$ has additional structure, namely a time reversal symmetry.

Assume that the vector field $f_{\alpha}$ admits a time reversing symmetry (For a survey see [17].) $\mathscr{S}_{\alpha}: V_{\alpha} \rightarrow V_{\alpha}$, i.e.

$$
\frac{d}{d t}\left(\mathscr{S}_{\alpha} \circ x_{\alpha}\right)=f_{\alpha}\left(\mathscr{S}_{\alpha} \circ x_{\alpha}\right) \Leftrightarrow \dot{x}_{\alpha}=f_{\alpha}\left(x_{\alpha}\right)
$$

that is also an involution: $\mathscr{S}_{\alpha} \circ \mathscr{S}_{\alpha}=i d$. This implies $\mathscr{S}_{\alpha} \circ f_{\alpha}^{t} \circ \mathscr{S}_{\alpha}=f_{\alpha}^{-t}$. We now investigate a composition of two partial return maps, $x_{\alpha_{1}}=\mathscr{S}_{\alpha} \circ r_{\alpha}^{\beta}\left(x_{\alpha_{0}}\right)$, followed by $x_{\alpha_{2}}=\mathscr{S}_{\alpha} \circ r_{\alpha}^{\beta}\left(x_{\alpha_{1}}\right)$. If the functional identity $t_{\alpha_{1}}^{\beta}=t_{\alpha_{0}}^{\beta}$ holds, then

$$
\begin{aligned}
\mathscr{S}_{\alpha} \circ r_{\alpha}^{\beta} \circ \mathscr{S}_{\alpha} \circ r_{\alpha}^{\beta}=\mathscr{S}_{\alpha} \circ f_{\alpha}^{t^{\beta}} \circ \mathscr{S}_{\alpha} \circ f_{\alpha}^{t^{\beta}}= & =(2) \\
f_{\alpha}^{-t^{\beta}} \circ f_{\alpha}^{t_{0}} & =i d
\end{aligned}
$$

i.e. $\mathscr{S}_{\alpha} \circ r_{\alpha}^{\beta}$ is also an involution. A sufficient condition for $t_{\alpha_{1}}^{\beta}=t_{\alpha_{0}}^{\beta}$ is given by

$$
\begin{aligned}
& \left(h_{\alpha}^{\beta}\left(f_{\alpha}^{t_{\alpha_{0}}^{\beta}}\left(x_{\alpha_{1}}\right), x_{\alpha_{1}}, t_{\alpha_{0}}^{\beta}\right)=0 \Leftrightarrow\right. \\
& \left.h_{\alpha}^{\beta}\left(f_{\alpha}^{t_{\alpha_{0}}^{\beta}}\left(x_{\alpha_{0}}\right), x_{\alpha_{0}}, t_{\alpha_{0}}^{\beta}\right)=0\right) \Rightarrow t_{\alpha_{1}}^{\beta}=t_{\alpha_{0}}^{\beta},
\end{aligned}
$$

or more explicitly

$$
\begin{aligned}
& \left(h_{\alpha}^{\beta}\left(f_{\alpha}^{t_{\alpha_{0}}^{\beta}} \circ S_{\alpha} \circ f_{\alpha}^{t_{\alpha_{0}}^{\beta}}\left(x_{\alpha_{0}}\right), S_{\alpha} \circ f_{\alpha}^{t^{\beta}}\left(x_{\alpha_{0}}\right), t_{\alpha_{0}}^{\beta}\right)=\right. \\
& \left.h_{\alpha}^{\beta}\left(S_{\alpha}\left(x_{\alpha_{0}}\right), S_{\alpha} \circ f_{\alpha}^{t_{\alpha_{0}}^{\beta}}\left(x_{\alpha_{0}}\right), t_{\alpha_{0}}^{\beta}\right)=0\right) \\
& \Leftrightarrow\left(h_{\alpha}^{\beta}\left(f_{\alpha}^{t_{\alpha_{0}}^{\beta}}\left(x_{\alpha_{0}}\right), x_{\alpha_{0}}, t_{\alpha_{0}}^{\beta}\right)=0\right)
\end{aligned}
$$

which can be considered an invariance of the threshold function under the partial return map $\mathscr{S}_{\alpha} \circ r_{\alpha}^{\beta}: x_{\alpha_{0}} \mapsto S_{\alpha}$ 。

${ }^{1}$ Note that this definition generalizes [15], where $h_{\alpha}^{\beta}$ only depends on $f_{\alpha}^{t}\left(x_{\alpha_{0}}\right)$. $r_{\alpha}^{\beta}\left(x_{\alpha_{0}}\right)$. This essentially checks that the threshold function $h_{\alpha}^{\beta}$ "preserves" the time reversal symmetry of $f_{\alpha}$.

Given that $\mathscr{S}_{\alpha} \circ r_{\alpha}^{\beta} \circ \mathscr{S}_{\alpha} \circ r_{\alpha}^{\beta}=i d$ we obtain

$$
\begin{aligned}
& D\left(\mathscr{S}_{\alpha} \circ r_{\alpha}^{\beta} \circ \mathscr{S}_{\alpha} \circ r_{\alpha}^{\beta}\right)=\mathbf{1}_{n \times n} \\
& =D \mathscr{S}_{\alpha}\left(r_{\alpha}^{\beta} \circ \mathscr{S}_{\alpha} \circ r_{\alpha}^{\beta}\right) \cdot D r_{\alpha}^{\beta}\left(\mathscr{S}_{\alpha} \circ r_{\alpha}^{\beta}\right) \cdot D \mathscr{S}_{\alpha}\left(r_{\alpha}^{\beta}\right) \cdot D r_{\alpha}^{\beta}
\end{aligned}
$$

Next, we call a trajectory on a chart $V_{\alpha}$ pseudosymmetric if its initial condition, $\bar{x}_{\alpha}$, is a fixed point of the partial return map $\mathscr{S}_{\alpha} \circ r_{\alpha}^{\beta}$, i.e. $\mathscr{S}_{\alpha} \circ r_{\alpha}^{\beta}\left(\bar{x}_{\alpha}\right)=\bar{x}_{\alpha}$. Evaluation of expression (5) at such a fixed point then allows us to determine the square of the determinant of the Jacobian of $r_{\alpha}^{\beta}$ (the monodromy matrix):

$$
\begin{aligned}
\left(D \mathscr{S}_{\alpha}\left(r_{\alpha}^{\beta}\left(\bar{x}_{\alpha}\right)\right) \cdot D r_{\alpha}^{\beta}\left(\bar{x}_{\alpha}\right)\right)^{2} & =\mathbf{1}_{n \times n} \\
\operatorname{det}^{2}\left(D \mathscr{S}_{\alpha}\left(r_{\alpha}^{\beta}\left(\bar{x}_{\alpha}\right)\right) \cdot D r_{\alpha}^{\beta}\left(\bar{x}_{\alpha}\right)\right) & =1
\end{aligned}
$$

If $\mathscr{S}_{\alpha}$ is linear, as is the case in all of the examples in this paper, then $\operatorname{det}^{2}\left(D \mathscr{S}_{\alpha}\right)=1$ and therefore

$$
\operatorname{det}^{2}\left(D r_{\alpha}^{\beta}\left(\bar{x}_{\alpha}\right)\right)=1
$$

Hence if a periodic orbit described by a fixed point of the full return map $\mathscr{R}$ is composed of pseudosymmetric orbits on $V_{\alpha}$ and conditions (3) hold on each chart, the full return map $\mathscr{R}$ is volume-preserving at this fixed point. If conditions (3) do not hold or if periodic orbits of the full return map are not composed of pseudosymmetric orbits, no conclusions can be drawn from this argument. Note that here "volume" is defined with respect to the state variables chosen, and in general is not a phase space volume, and that the computation (at the fixed point) is local.

\section{APPLICATION TO HYBRID MODELS OF LEGGED LOCOMOTION}

\section{A. SLIP with pitching}

The SLIP model consists of a rigid body of mass $m$ and moment of inertia $I$ with a massless springy leg attached to an unactuated hip joint which is a distance $d$ away from the center of mass (for details see [8]). A full stride consists of a stance phase with the foothold fixed and the leg under compression, and a flight phase in which the body describes a ballistic trajectory. Hence there are two vector fields $f_{1}$ (for stance) and $f_{2}$ (for flight) and the return map can be written as $\mathscr{R}=\mathscr{T}_{2}^{1} \circ r_{2}^{1} \circ \mathscr{T}_{1}^{2} \circ r_{1}^{2}$. We assume that a periodic orbit of period one is composed of pseudosymmetric stance and flight phases. ${ }^{2}$

\footnotetext{
${ }^{2}$ This was proven in [8] for SLIP without pitching dynamics and without gravity in stance.
} 


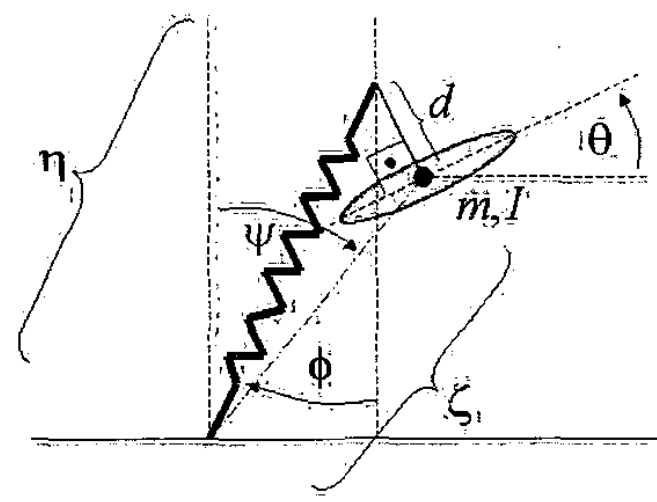

Fig. 1. Parametrization of SLIP with pitching dynamics.

1) Stance phase of SLIP with pitching: The equations of motion that describe the stance phase of SLIP with mass $m$ and moment of inertia $l$ read in the conventions of Fig. 1 (see also [8]):

$$
\begin{aligned}
\ddot{\zeta} & =\zeta \dot{\psi}^{2}-g \cos \psi-\frac{\partial_{\eta} V(\eta)}{m \eta}(\zeta+d \cos (\psi+\theta)) \\
\zeta \ddot{\psi} & =-2 \zeta \dot{\psi}+g \sin \psi+d \frac{\partial_{\eta} V(\eta)}{m \eta}(\sin (\psi+\theta)) \\
\ddot{\theta} & =d \zeta \frac{\partial_{\eta} V(\eta)}{\eta I} \sin (\psi+\theta)
\end{aligned}
$$

with spring potential $V(\eta)$ where the compressed spring length $\eta=\sqrt{d^{2}+\zeta^{2}+2 d \zeta \cos (\psi+\theta)} \leq \eta_{0}$, the relaxed spring length being denoted by $\eta_{0}$. The distance between the 'hip' pivot and the mass center is $d$ and all joints, including the toe-ground pivot, are assumed frictionless and moment-free.

The involutive time reversing symmetry $\mathscr{S}_{1}$ of (6) acting on $x_{1}=(\zeta \psi \theta \dot{\zeta} \dot{\psi} \dot{\theta})^{\top}$ is given by the linear map

$$
\mathscr{S}_{1}=\operatorname{diag}(1,-1,-1,-1,1,1) .
$$

Under $\mathscr{S}_{1}$, the spring length $\eta$ remains invariant: $\mathscr{S}_{1}(\eta)=$ $\eta$. Transitions to flight occur when the spring length has reached its rest length $\eta_{0}$ which is also the initial spring length at the beginning of stance. Hence the threshold function can be written as $h_{1}^{2}\left(x_{1}(t), x_{1_{0}}, t\right)=\eta^{2}(t)-\eta_{0}^{2}$. Then the partial return map $\mathscr{S}_{1} \circ r_{1}^{2}$ is volume-preserving at a fixed point, because (4) holds:

$$
\begin{aligned}
h_{1}^{2}\left(x_{1}\left(t_{1_{0}}^{2}\right), x_{1_{0}}, t_{1_{0}}^{2}\right) & =\eta^{2}\left(t_{1_{0}}^{2}\right)-\eta_{0}^{2}=0 \\
\Leftrightarrow h_{1}^{2}\left(\mathscr{S}_{1} \cdot x_{1_{0}}, \mathscr{S}_{1} \cdot x_{1}\left(t_{1_{0}}^{2}\right), t_{1_{0}}^{2}\right) & =\eta_{0}^{2}-\eta^{2}\left(t_{1_{0}}^{2}\right)=0
\end{aligned}
$$

This result is independent of the specific form of the spring potential $V(\eta)$. For $d=0$, the two-dimensional
SLIP system describing a point mass on a massless spring is recovered.

2) Flight phase of SLIP with pitching: The equation of motion of the center of mass that describe the flight phase of SLIP read

$$
\ddot{y}=0, \quad \ddot{z}=-g, \quad \ddot{\theta}=0
$$

where the $z$-axis points vertically upwards, the $y$-axis points in a horizontal direction, and $\theta$ denotes the SLIP body's pitching angle with respect to the horizontal. The linear involutive time reversing symmetry $\mathscr{S}_{2}$ of $(8)$ acting on $x_{2}=\left(\begin{array}{lll}y z & \theta \dot{y} \dot{z} \dot{\theta}\end{array}\right)^{\top}$ is given by

$$
\mathscr{S}_{2}=\operatorname{diag}( \pm 1,1,-1, \mp 1,-1,1) .
$$

Here, the sign ambiguity in $\theta$ was resolved by matching $\theta$ 's transformation to that of the stance phase (7). The transformation law of $y$ under $\mathscr{S}_{2}$ is not needed in subsequent calculations ${ }^{3}$ and is left unresolved. Hence from now on, we work with a reduced state vector $x_{2} \rightarrow$ $(z \theta \dot{z} \dot{\theta})^{\top}$.

The simplicity of the equations of motion (8) allows us to explicitly compute the determinant of the monodromy matrix of $\mathscr{S}_{2} \circ r_{2}^{1}$ for a given leg recirculation scheme. Therefore the application of the formalism of Sec. 2, which only provides a sufficient condition for volumepreservation, but not for non-preservation, is relegated to the appendix.

The equations of motion for the $z$ and $\theta$ coordinates can be explicitly solved and read in dimensionless variables:

$$
\begin{aligned}
\tilde{z}(\tilde{t}) & =\tilde{z}_{0}+\dot{\tilde{z}}_{0} \tilde{t}-\frac{\tilde{t}^{2}}{2} \\
\dot{\bar{z}}(\tilde{t}) & =\dot{\tilde{z}}_{0}-\tilde{t} \\
\tilde{\theta}(\tilde{t}) & =\tilde{\theta}_{0}+\dot{\tilde{\theta}}_{0} \tilde{t} \\
\dot{\tilde{\theta}}(\tilde{t}) & =\dot{\tilde{\theta}}_{0}
\end{aligned}
$$

with $\tilde{t}=t \sqrt{\frac{g}{\eta_{0}}}, \tilde{z}=\frac{z}{\eta_{0}}, \dot{\tilde{z}}=\frac{\dot{z}}{\sqrt{\eta_{0} g}}, \tilde{\theta}_{0}=\theta_{0}$, and $\dot{\tilde{\theta}}=\dot{\theta} \sqrt{\frac{\eta_{0}}{g}}$. We now want to explore different strategies to position the leg during flight. Since the leg is assumed massless, any leg angle trajectory $\phi\left(\tilde{t}, \tilde{x}_{2_{0}}\right)$ where $\phi$ is defined in Fig. 1 can be commanded.

The threshold function $h_{2}^{!}$for a recirculating leg reads in dimensionless variables

$$
h_{2}^{1}\left(\tilde{x}_{2_{0}}(\tilde{t}), \tilde{x}_{2_{0}}, \tilde{t}\right)=\bar{z}(\tilde{t})+\tilde{d} \cos (\tilde{\theta}(\tilde{t}))-\cos \left(\phi\left(\tilde{t}, \tilde{x}_{2_{0}}\right)\right)
$$

where $\tilde{d}=\frac{d}{\eta_{0}}$. Setting (11) to zero determines the time from leg liftoff $\left(\tilde{t}_{L O}=0\right.$ ) to leg touchdown $\tilde{t}_{T D}:=\tilde{t}_{2_{0}}^{1}$, for which in general a closed form solution does not exist. Then the flow map $r_{2}^{1}$ takes the dimensionless state vector $\vec{x}_{2_{0}}=\left(\begin{array}{llll}\tilde{z} & \dot{\tilde{z}} & \tilde{\theta} & \dot{\tilde{\theta}}\end{array}\right)^{\top}$ from its value at leg liftoff to that at

${ }^{3} \dot{y}$ is determined by conservation of energy and $y$ is not a periodic variable. 
touchdown: $r_{2}^{1}\left(\tilde{x}_{2_{0}}\right)=\tilde{x}_{2}\left(\tilde{t}_{T D}\right)$. A fixed point of a pseudosymmetric flight trajectory satisfies $\overline{\tilde{x}}_{2_{0}}=\mathscr{S}_{2} \circ r_{2}^{\prime}\left(\overline{\tilde{x}}_{2_{0}}\right)$.

The determinant of the monodromy matrix of $r_{2}^{1}$ can easily be computed from the flight trajectory expressions (10), bearing in mind that the flight time $\tilde{t}_{T D}$ also depends on the initial conditions:

$$
\operatorname{det}\left(D r_{2}^{1}\right)=1-\partial_{\dot{\bar{z}}_{0}} \tilde{t}_{T D}+\dot{\tilde{z}}_{0} \partial_{\tilde{z}_{0}} \tilde{t}_{T D}+\dot{\tilde{\theta}}_{0} \partial_{\tilde{\theta}_{0}} \tilde{t}_{T D}
$$

In this expression, the leading term 1 is a consequence of Liouville's theorem, because $\bar{z}$ and $\dot{\tilde{z}}$ and $\tilde{\theta}$ and $\dot{\tilde{\theta}}$ are canonically conjugate up to a trivial rescaling, whereas the remaining terms make the non-applicability of Liouville's theorem to this hybrid system with a state-dependent threshold function (11) explicit. Hence using implicit differentiation of (11) the determinant can be written in terms of partial derivatives of $\phi\left(\tilde{t}, \tilde{x}_{2_{0}}\right)$ :

$$
\operatorname{det}\left(D r_{2}^{1}\left(\tilde{x}_{2_{0}}\right)\right)=1+\left.\frac{\Delta_{2}^{1 \text { num }}}{\Delta_{2}^{1} \operatorname{den}}\right|_{i=\tilde{t}_{T D}}
$$

with

$$
\begin{aligned}
& \Delta_{2}^{1 \text { num }}=\sin \left(\phi\left(\tilde{t}, \tilde{x}_{2_{0}}\right)\right) \cdot \\
& \quad\left(\partial_{\tilde{z}_{0}} \phi\left(\tilde{t}, \tilde{x}_{2_{0}}\right)-\dot{\tilde{z}}_{0} \partial_{\tilde{z}_{0}} \phi\left(\tilde{t}, \tilde{x}_{2_{0}}\right)-\dot{\tilde{\theta}}_{0} \partial_{\tilde{\theta}_{0}} \phi\left(\tilde{t}, \tilde{x}_{2_{0}}\right)\right) \\
& \quad+\tilde{t}-\dot{z}_{0}+\tilde{d} \sin \left(\tilde{\theta}_{0}\right) \dot{\tilde{\theta}}_{0} \\
& \Delta_{2}^{1 \mathrm{den}}=\sin \left(\phi\left(\tilde{t}, \tilde{x}_{2_{0}}\right)\right) \partial_{\hat{t}} \phi\left(\tilde{t}, \tilde{x}_{2_{0}}\right)-\tilde{t}+\dot{\tilde{z}}_{0}-\tilde{d} \sin \left(\tilde{\theta}_{0}\right) \dot{\tilde{\theta}}_{0} .
\end{aligned}
$$

Albeit $\tilde{t}_{T D}$ cannot be computed explicitly in general, $\tilde{t}_{T D}=$ $2 \dot{z}_{0}$ at a symmetric (period-1) fixed point of $\mathscr{S}_{2} \circ r_{2}^{1}$, $\sin \left(\phi\left(\tilde{t}_{T D}, \tilde{x}_{2_{0}}\right)\right)=-\sqrt{1-\left(\tilde{z}_{0}+\tilde{d} \cos \left(\tilde{\theta}_{0}\right)\right)^{2}}$ and $\theta\left(\tilde{t}_{T D}\right)=$ $-\tilde{\theta}_{0}$. The eigenvalues of the partial return map $\mathscr{S}_{2} \circ r_{2}^{1}$ at such a fixed point are $\lambda_{1}=1$ (vertical energy), $\lambda_{2}=1$ (rotational energy), $\lambda_{3}=-1$, and $\lambda_{4}=-\operatorname{det}\left(D r_{2}^{1}\left(\overline{\tilde{x}}_{2_{0}}\right)\right.$ ). It must be emphasized, however, that the eigenvalues of this partial retum map are not equal to the eigenvalues of the total return map $\mathscr{R}$ at the fixed point. In particular, the eigenvalues of $\mathscr{R}$ can be complex, as in Fig. 2, below.

Setting (13) to 1 yields a partial differential equation for leg recirculation schemes $\phi\left(\tilde{t}, \tilde{x}_{2_{0}}\right)$ that are volume preserving. In the following, for simplicity, we apply formula (13) to different leg recirculation schemes for SLIP without pitching dynamics, i.e. $\tilde{d}=0$ and no $\tilde{\theta}, \dot{\theta}$ dependence. The investigation of leg recirculation strategies of SLIP with pitching dynamics will be detailed in [10]. For SLIP without pitching, the determinant at the fixed point simplifies to

$$
\begin{aligned}
& \operatorname{det}\left(D r_{2}^{1}\left(\tilde{x}_{2_{0}}\right)\right)=1+ \\
& \left.\frac{\sin \left(\phi\left(\tilde{t}, \tilde{x}_{2_{0}}\right)\right)\left(\partial_{\dot{z}_{0}} \phi\left(\tilde{t}, \tilde{x}_{2_{0}}\right)-\dot{\tilde{z}}_{0} \partial_{\tilde{z}_{0}} \phi\left(\tilde{t}, \tilde{x}_{2_{0}}\right)\right)+\tilde{t}-\dot{z}_{0}}{\sin \left(\phi\left(\tilde{t}, \tilde{x}_{2_{0}}\right)\right) \partial_{\tilde{t}} \phi\left(\tilde{t}, \tilde{x}_{2_{0}}\right)-\tilde{t}+\dot{\tilde{z}}_{0}}\right|_{\tilde{t}=\tilde{t}_{T D}}
\end{aligned}
$$

3) Analysis of Recirculation Strategies: Consider now the following family of leg recirculation schemes

$$
\phi\left(\tilde{t}, \tilde{x}_{2_{0}}\right)=k \arccos \left(\tilde{z}_{0}\right)+\alpha\left(\tilde{t}-l \dot{z}_{0}\right): k, l>0
$$

For $k \neq 0$ leg recirculation starts at an angle proportional to the leg liftoff angle; if $l=1$, a certain angular trajectory is specified starting at apex (see the leg retraction scheme in [9]).

The application of the partial return map $\mathscr{S}_{2} \circ r_{2}^{1}$ on $\tilde{x}_{2_{0}}$ in the threshold function $h_{2}^{1}$ (4) in order to determine for which parameters of the leg recirculation schemes (15) volume is preserved is relegated to the appendix. Instead we proceed by explicitly computing $\operatorname{det}\left(\operatorname{Dr}_{2}^{1}\left(\tilde{x}_{2_{0}}\right)\right)$. With the angular trajectory (15), the determinant becomes

$$
\begin{aligned}
& \operatorname{det}\left(D r_{2}^{1}\left(\tilde{x}_{2_{0}}\right)\right)=1+ \\
& \left.\frac{\sin \left(\phi\left(\tilde{t}, \tilde{x}_{2_{0}}\right)\right)\left(-l \dot{\alpha}\left(\tilde{t}-l \dot{\tilde{z}}_{0}\right)-\frac{k \dot{z}_{0}}{\sqrt{1-\bar{z}_{0}^{2}}}\right)+\tilde{t}-\dot{\tilde{z}}_{0}}{\sin \left(\phi\left(\tilde{t}, \tilde{x}_{2_{0}}\right)\right) \dot{\alpha}\left(\tilde{t}-l \dot{\bar{z}}_{0}\right)-\tilde{t}+\dot{\tilde{z}}_{0}}\right|_{\tilde{i}=\tilde{T}_{T D}}
\end{aligned}
$$

Hence for different leg angle protocols we obtain

1) Constant leg touchdown angle protocol: $k=l=$ $0, \alpha=2 \pi-\beta \Rightarrow \operatorname{det}\left(\operatorname{Dr}_{2}^{1}\left(\tilde{x}_{2_{0}}\right)\right)=0$. The twodimensional monodromy matrix has rank one for all $\tilde{t}_{T D}$, and the return map becomes one-dimensional. In [7] this return map was parametrized by apex height, whereas in [8] the angle of the touchdown velocity was chosen. No information about the behavior of this lower-dimensional return map can be obtained from this argument.

2) Leg retraction [9]: $k=0, l=1$, and $\alpha\left(\tilde{t}-\dot{z}_{0}\right)=$ $\alpha_{A}+\tilde{\omega}\left(\tilde{t}-\dot{\bar{z}}_{0}\right)$ where $\alpha_{A}$ is a constant angle and $\tilde{\omega}=$ $\omega \sqrt{\frac{\eta_{0}}{g}}$ is a constant dimensionless angular velocity. Then again $\operatorname{det}\left(\operatorname{Dr}_{2}^{1}\left(\tilde{x}_{2_{0}}\right)\right)=0$ and the behavior of the remaining one-dimensional return map cannot be determined from this argument.

3) Leg recirculation (starting at leg liftoff): $l=0$, $k>0$, and $\alpha(\tilde{t})=\alpha_{A}+\tilde{\omega} \tilde{t}$. This exemplifies the fast rotation phase of the open loop policy used by RHex [1], although a full analysis is beyond the scope of the present paper. Then the determinant of the monodromy matrix at the symmetric fixed point becomes:

$$
\begin{aligned}
\operatorname{det}\left(\operatorname{Dr}_{2}^{1}\left(\overline{\tilde{x}}_{2_{0}}\right)\right)= & 1-\frac{\dot{\tilde{z}}_{0}(1-k)}{\dot{\tilde{z}}_{0}+\tilde{\omega} \sqrt{1-\tilde{z}_{0}^{2}}} \\
= & \left\{\begin{array}{ccc}
<1 & : & 0<k<1 \\
1 & : & k=1 \\
>1 & : & k>1
\end{array}\right.
\end{aligned}
$$

In order to illustrate the predictive power of (17), we numerically approximate the determinant $\operatorname{det}(D \mathscr{R}(\bar{x}))$ of 
the full retum map for fixed SLIP parameters $\tilde{E}=\frac{E}{m g \eta_{0}}=$ 2.1, $\gamma=\frac{\kappa \eta_{0}}{m g}=13$, and fixed recirculation parameters $\alpha_{A}=\pi, \tilde{\omega}=14$ for different $k \in\{1 / 6,0.5,1,2,3.3\}$. Here, $E$ is the total energy of the system and the spring potential is $V(\eta)=(\kappa / 2)\left(\eta-\eta_{0}\right)^{2}$. We then compare these values to the values of the determinant obtained by inserting the numerically determined fixed points $\overrightarrow{\tilde{x}}_{2_{0}}=$ $\left(\bar{z}_{0} \overline{\tilde{z}}_{0}\right)^{\top}$ into (17). The determinants obtained in those two different ways are plotted in Fig. 2a and agree to a high precision $\left(\left|\operatorname{det}(D \mathscr{R}(\bar{x}))-\operatorname{det}\left(D r_{2}^{1}\left(\overline{\tilde{x}}_{2_{0}}\right)\right)\right|<10^{-7}\right)$. Barring an improbable numerical cancellation between stance and flight phase dynamics, this also demonstrates that the SLIP's stance phase is volume preserving. ${ }^{4}$ In Figs. 2b-d iterations of the return map in $\left(\overline{\tilde{z}}_{0} \overline{\bar{z}}_{0}\right)$-space are shown for $k \in\{1 / 6,1,3.3\}$ and initial conditions off the fixed point. The eigenvalues are complex conjugate pairs in all three cases. For $k=1 / 6$ the trajectory spirals towards the fixed point, as expected from a stable fixed point (Fig. 2c), for $k=1$ the trajectory is a deformed circle around the fixed point, ${ }^{5}$ indicating neutral stability (Fig. $2 \mathrm{~d}$ ), and for $k=3.3$ the trajectory spirals away from the fixed point, indicating instability (Fig. 2b).

\section{B. Lateral leg-spring model}

The lateral leg-spring (LLS) was introduced in [6]. We focus here on the three-degree-of-freedom version with pairs of 'virtual' elastic legs in intermittent contact with the ground. A full stride consists of two stance phases: a phase where the first elastic leg pivots around a "foothold" on one side of the rigid body, followed by a phase where the second elastic leg pivots around a "foothold" on the opposite side. See [6] for details. The equations of motion of both stance phases can be cast into the form (6) (with $g=0$ ). Hence the stance phases from leg touchdown to liftoff are volume preserving. They are related by a transition mapping $\mathscr{T}_{1}^{2}$ which maps the state at liftoff of the 1 . leg to the state at touchdown of the 2 . leg, and an analogous map $\mathscr{T}_{2}^{1}$. Thus the return map reads $\mathscr{R}=\mathscr{T}_{2}^{1} \circ r_{2}^{1} \circ \mathscr{T}_{1}^{2} \circ r_{1}^{2}$.

The dynamics of the LLS model can be described by four state variables $(v, \delta, \theta, \omega)$, where $v$ is the center of mass speed, $\delta$ is the angle between the body axis and the mass center velocity vector, $\theta$ is the angle between the body axis and an inertial frame and $\omega=\dot{\theta}$. In [6] these four variables are augmented by two fixed parameters: $\beta$,

\footnotetext{
${ }^{4}$ This is not true for approximations to the stance phase dynamics which violate the time reversing symmetry $\mathscr{S}_{1}$.

${ }^{5}$ Numerical evidence shows that closed curves persist in any neighborhood around the fixed point. Since the determinant away from the fixed point is not 1, the standard KAM theorem for 2-dimensional maps (see e.g. [18]) is not applicable here. However, the reverse time symmetry of the leg reciculation strategy in this example can be shown to entail the existence of Kolmogorov tori around the fixed point. This is, a consequence of a result on reversible systems [19, Theorem: 2.9 , for more details see [10].
}

the leg touchdown angle with respect to the body axis, and $l_{0}$, the relaxed leg length; and from the values of these six quantities at liftoff one can find the initial data for the next stance phase.

In these variables, the transition mapping $\mathscr{T}_{1}^{2}$ (omitting $l_{0}$ ) reads

$$
\mathscr{T}_{1}^{2}\left(\left(v_{n}^{L O} \delta_{n}^{L O} \theta_{n}^{L O} \omega_{n}^{L O} \beta_{n}^{L O}\right)\right)=\left(v_{n}^{L O} \delta_{n}^{L O} \theta_{n}^{L O} \omega_{n}^{L O} \beta\right)
$$

where $n$ stands for the $n$th stance phase, $L O$ for liftoff, and $\beta$ is held constant for all stance phases. If, as implicitly assumed for the SLIP treated above, $\beta$ (and/or $l_{0}$ ) are regarded as state variables rather than parameters, then since they are 'reset' to fixed values at touchdown, independent of their values at liftoff, the transition mapping $\mathscr{T}_{1}^{2}$ has rank four, volume is not preserved, and no deductions can be made regarding the reduced four-dimensional map. Restoring a nontrivial dynamical role to the variable $\beta$, for example, via a leg swing feedback strategy similar to (15), could lead to a non-degenerate mapping.

\section{CONCLUSIONS}

In this paper we used the example of the SLIP locomotion model to show how factored analysis of the return map may be a useful new tool in the stability analysis of hybrid Lagrangian systems. Specifically, we obtained a necessary condition for the asymptotic stability of SLIP in the presence of a leg recirculation strategy relevant to the operation of the robot RHex [1]. This condition is formulated in Sec. III.A.3 for a particular family of leg recirculation strategies as an exact algebraic expression despite the non-integrability of the SLIP system. Hence leg recirculation strategies that violate the above condition can be discarded without recourse to cumbersome numerical simulations. Application of this formalism to the robot RHex requires a more elaborate parametrization of leg recirculation schemes modeled after RHex's open loop controller.

This analysis can provide for the first time a partial explanation for the surprising self-stable behavior observed empirically in RHex. It also paves the way for a more principled investigation of detailed, biologically motivated leg placement strategies in the LLS model [6] which captures many aspects of cockroach locomotion [20].

\section{ACKNOWLEDGEMENTS}

This work is supported in part by DARPA/ONR Grant No0014-98-1-0747. Helpful discussions with R. Ghigliazza are gratefully acknowledged.

\section{APPENDIX: INVARIANCE OF THE THRESHOLD EQUATION FOR SLIP WITHOUT PITCHING}

In this appendix we show that the invariance of the threshold equation (11) under $S_{2} \circ r_{2}^{1}: \tilde{x}_{2_{0}} \mapsto S_{2} \circ f_{2}^{\tilde{i}_{T D}}\left(\bar{x}_{2_{0}}\right)$ 


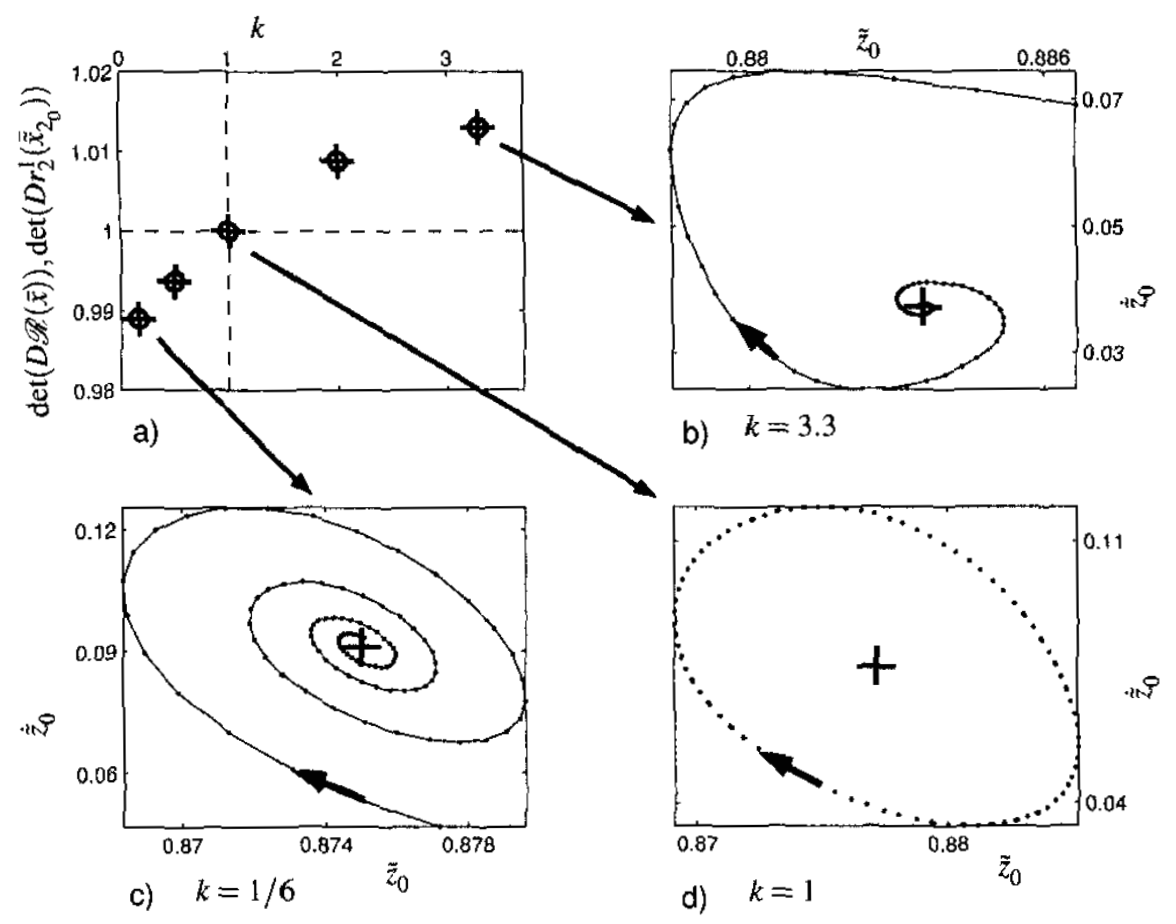

Fig. 2. a) Comparison of the numerically computed determinant $\operatorname{det}(D \mathscr{R}(\bar{x}))(+)$ of the retum map monodromy matrix to to the determinant $\operatorname{det}\left(D r_{2}^{1}\left(\overline{\bar{x}}_{2_{0}}\right)\right)$ (o) obtained by using the numerically determined fixed points in (17). b)-d) Trajectories around a fixed point. Because of slow convergence, only every 9 th iteration in plot $b$ ) and every 5 th itcration in plot c) is shown.

with $\tilde{x}_{2_{0}}=\left(\tilde{z}_{0} \dot{\tilde{z}}_{0}\right)^{\top}$ corresponds to $\left|\operatorname{det}\left(D r_{2}^{1}\right)\right|=1$ for the leg recirculation family (15). First we observe that

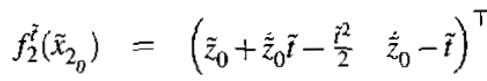

$$
\begin{aligned}
& \mathscr{S}_{2} \circ f_{2}^{\tilde{t}}\left(\tilde{x}_{2_{0}}\right)=\left(\tilde{z}_{0}+\dot{\tilde{z}}_{0} \tilde{t}-\frac{\tilde{t}^{2}}{2}-\left(\dot{z}_{0}-\tilde{t}\right)\right)^{\top} \\
& f_{2}^{\tilde{I}} \circ \mathscr{S}_{2} \circ f_{2}^{\tilde{t}}\left(\tilde{x}_{2_{0}}\right)=\left(\begin{array}{ll}
\tilde{z}_{0} & -\dot{\bar{z}}_{0}
\end{array}\right)^{\top}
\end{aligned}
$$

The threshold function $h_{2}^{1}\left(f_{2}^{\tilde{t}}\left(\tilde{x}_{2_{0}}\right), \tilde{x}_{2_{0}}, \tilde{t}\right)$ becomes

$$
\begin{array}{r}
\quad h_{2}^{1}\left(f_{2}^{\tilde{t}}\left(\tilde{x}_{2_{0}}\right), \tilde{x}_{2_{0}}, \tilde{t}\right)=\tilde{z}(\tilde{t})-\cos \left(\phi\left(\tilde{z}_{0}, \dot{z}_{0}, \tilde{t}\right)\right) \\
\text { with } \phi\left(\tilde{z}_{0}, \dot{z}_{0}, \tilde{t}\right)=k \arccos \left(\tilde{z}_{0}\right)+\alpha\left(\tilde{t}-l \dot{z}_{0}\right) .
\end{array}
$$

Then

$$
\begin{aligned}
h_{2}^{1}\left(f_{2}^{\tilde{t}_{T D}} \circ \mathscr{S}_{2} \circ f_{2}^{\tilde{T}_{T D}}\left(\tilde{x}_{2_{0}}\right), \mathscr{S}_{2} \circ f_{2}^{\tilde{t}_{T D}}\left(\tilde{x}_{2_{0}}\right), \tilde{t}_{T D}\right)= & \\
\tilde{z}_{0}-\cos \left(k \arccos \left(\tilde{z}_{0}+\dot{\tilde{z}}_{0} \tilde{t}_{T D}-\frac{\tilde{t}_{T D}^{2}}{2}\right)\right. & \\
\left.+\alpha\left(\tilde{t}_{T D}+l\left(\dot{\bar{z}}_{0}-\tilde{t}_{T D}\right)\right)\right) & =0
\end{aligned}
$$

For a solution of this equation with the leg recirculating only once during flight $\phi\left(\tilde{z}_{0}^{\prime}, \dot{z}_{0}, \tilde{t}_{T D}\right) \in\left(\frac{3}{2} \pi, 2 \pi\right)$. This must be taken into account when inverting the cosine:

$$
\begin{array}{ll} 
& \arccos \left(\tilde{z}_{0}\right)=-\left(k \arccos \left(\tilde{z}_{0}+\dot{\tilde{z}}_{0} \tilde{t}_{T D}-\frac{\tilde{t}_{T D}^{2}}{2}\right)\right. \\
& \left.+\alpha\left(\tilde{t}_{T D}+l\left(\dot{\tilde{z}}_{0}-\tilde{t}_{T D}\right)\right)\right)+2 \pi \\
\Leftrightarrow \quad & \cos \left(k \arccos \left(\tilde{z}\left(\tilde{t}_{T D}\right)\right)\right)-\cos \left(\arccos \left(\tilde{z}_{0}\right)\right. \\
& \left.+\alpha\left(\tilde{t}_{T D}+l\left(\dot{z}_{0}-\tilde{t}_{T D}\right)\right)\right)=0 \\
k=1, l=0 & \tilde{z}\left(\tilde{t}_{T D}\right)-\cos \left(\arccos \left(\tilde{z}_{0}\right)+\alpha\left(\tilde{t}_{T D}\right)\right)=0
\end{array}
$$

For $k=1$ and $l=0$ this does reduce to the original threshold function (18) and we conclude that $\left|\operatorname{det}\left(D r_{2}^{1}\right)\right|=$ 1 , as was explicitly derived in (17). For other values of $k$ and $l$ this does not in general reduce to (18), although we have not ruled out that for specific values of $k$ and $I$ and a specific form of $\alpha$ the original threshold function (18) is recovered.

\section{REFERENCES}

[1] U. Saranli, M. Buehler, and D.E. Koditschek. Rhex: A simple and highly mobile hexapod robot. The In- 
ternational Journal of Robotics Research, 20(7):616$631,2001$.

[2] R. Altendorfer, N. Moore, H. Komsuoğlu, M. Buehler, H.B. Brown Jr., D. McMordie, U. Saranli, R. Full, and D.E. Koditschek. Rhex: A biologically inspired hexapod runner. Autonomous Robots, 11:207-213, 2001.

[3] R. Blickhan and R. Full. Similarity in multilegged locomotion: Bouncing like a monopode. J. Comp. Physiol., A(173):509-517, 1993.

[4] M.H. Raibert. Legged Robots that Balance. MIT Press, Cambridge, MA, 1986.

[5] T. Kubow and R. Full. The role of the mechanical system in control: a hypothesis of self-stabilization in hexapedal runners. Philosophical Transactions of the Royal Society of London Series B - Biological Sciences, 354(1385):849-861, 1999.

[6] J. Schmitt and P. Holmes. Mechanical models for insect locomotion: dynamics and stability in the horizontal plane I. Theory. Biological Cybernetics, 83:501-515, 2000.

[7] A. Seyfarth, H. Geyer, M. Günther, and R. Blickhan. A movement criterion for running. Journal of Biomechanics, 35:649-655, 2002.

[8] R. M. Ghigliazza, R. Altendorfer, P. Holmes, and D. E. Koditschek. A simply stabilized running model. to appear in SIAM Journal on Applied Dynamical Systems, 2003.

[9] A. Seyfarth, H. Geyer, R. Blickhan, and H. Herr. Does leg retraction simplify control in running? In IV. World Congress of Biomechanics, Calgary, Canada, 2002.

[10] R. Altendorfer, R. M. Ghigliazza, P. Holmes, and D. E. Koditschek. Hopping on a springy leg: "low attention" feedback control. In preparation, 2003.
[11] H.M. Herr and T.A. McMahon. A trotting horse model. International Journal of Robotics Research, 19 (6):566-581, 2000.

[12] P. Holmes. Poincaré, celestial mechanics, dynamical systems theory and "chaos". Phys. Rep., 193(3):137$163,1990$.

[13] F. Scheck. Mechanics: from Newton's laws to deterministic chaos. Springer-Verlag, Berlin, 1999. Third edition.

[14] A. Ruina. Nonholonomic stability aspects of piecewise holonomic systems. Reports on mathematical physics, 42 (1-2):91-100, 1998.

[15] J. Guckenheimer and S. Johnson. Planar hybrid systems. In Hybrid systems II: Lecture notes in computer science, pages 202-225. Springer-Verlag, Berlin, 1995.

[16] M.J. Coleman and P. Holmes. Motions and stability of a piecewise holonomic system: the discrete Chaplygin sleigh. Regular and chaotic dynamics, 4(2):55-77, 1999.

[17] J.S.W. Lamb and J.A.G. Roberts. Time-reversal symmetry in dynamical systems: A survey. Physica D, 112:1-39, 1998.

[18] J. Moser. Stable and random motions in dynamical systems. Princeton University Press, 1973.

[19] M.B. Sevryuk. Reversible Systems. Number 1211 in Lecture notes in mathematics. Springer-Verlag, 1986.

[20] J. Schmitt, M. Garcia, R. Razo, P. Holmes, and R.J. Full. Dynamics and stability of legged locomotion in the horizontal plane: A test case using insects. Biological Cybernetics, 86 (5):343-353, 2002. 